\title{
Research on the Architecture of Enterprise Knowledge Network Construction
}

\author{
Bingfeng Liu and Guojing He \\ School of Management and Economics, Jingdezhen Ceramic Institute, Jiangxi Province, China \\ 234074489@qq.com
}

\begin{abstract}
Keywords: Enterprise knowledge; Knowledge network; Network construction; Architecture of enterprise knowledge; Information technology
\end{abstract}

\begin{abstract}
For the enterprise knowledge network, we should give full play to the role of knowledge network in knowledge sharing, knowledge reuse and knowledge innovation. The construction of knowledge network is based on the development of enterprise knowledge management and knowledge engineering. This paper first discusses the existing problems of enterprise knowledge management strategy, analysis of the specific needs of enterprise knowledge network construction and the construction of the target, resulting from the establishment, operation and orderly and knowledge integration in three aspects of the proposed top-level architecture framework of enterprise knowledge network construction; and respectively describes the construction and operation process of knowledge network; the final analysis the framework of the relationship between the needs of key technologies.
\end{abstract}

\section{Introduction}

The development of a new generation of information technology, computer hardware and data storage capacity, computing capability, rapid development of Internet technology, Web2. 0 cloud computing technology, big data technology, manufacturing industry, especially large and complex product manufacturing enterprises must keep pace with the trend of technological development, and constantly improve the technical system of enterprises. With the development of information technology, enterprises have been able to reasonably control the accumulated intellectual assets, clarify the distribution of knowledge, and give full play to the role of knowledge management and knowledge reuse.

In addition to technical support, the strategic thinking of enterprise knowledge management is equally important. Many managers believe that once the establishment of a knowledge management system, equipped with the corresponding knowledge base, knowledge service platform, the effect of knowledge management can automatically change. In fact, even if there is such a system, if not considered from the system, no effective means to find the knowledge sharing, application, innovation, evaluation and supervision activities to form an effective closed-loop operation system, knowledge management of enterprises is also very difficult to reach the ideal effect, now both in academia and in the enterprise management practice this little effective closed-loop system. From the aspects of technology, effect, income, security and evaluation, we can promote the development of enterprise knowledge management. In this paper, the construction of enterprise knowledge network system should be taken into consideration in order to solve the above problems.

\section{The Goal of Constructing Enterprise Knowledge Network}

Starting from the characteristics of knowledge network, combined with the enterprise actual business to achieve the following goals: the structure characteristics of the knowledge network organization requirements should be effectively scattered in every corner of the enterprise organizational knowledge resources become clear network system; dynamic characteristics of knowledge network of the enterprise shall keep the operation and optimization of the dynamic process of knowledge network construction. The content of knowledge network evolution with the change of time, environment and demand; from 
the knowledge network, knowledge integration goal-driven characteristics is not a simple construction of knowledge base, expert knowledge, but should guide and push function, to provide reasonable support for the user to solve the problem of knowledge. In addition, the construction of knowledge network needs to solve the problems of information ethics, intellectual property rights and so on.

The specific work shall be business and enterprise staff's knowledge network combination: to solve a large number of heterogeneous data cannot be used to solve the problem; knowledge discovery and a large number of repetitive work in the sharing of knowledge to solve problems; excessive and disorderly, resulting in knowledge discovery and sharing efficiency and declared use is not high, a lot of tacit knowledge sharing problem; solve the enthusiasm of employees to participate in knowledge sharing and knowledge ordering is not high, to help enterprises to create a collaborative information environment, solve the ordering of the enterprise knowledge (such as knowledge ontology base) method is applied in high cost without buying time in question.

Combining the information technology and management technology and design technology, the formation of the employees to actively and responsibly participate in knowledge construction and maintenance of the network business atmosphere, make the knowledge network orderly development into the development of a virtuous cycle of knowledge network system of positive feedback, provide a platform for knowledge sharing, support staff, initiative actively share their knowledge, the organization of individual knowledge and tacit knowledge; provide a knowledge resource ordering platform, improve the knowledge search efficiency, facilitate knowledge reuse; provide a prediction technology platform, collaborative technical support staff forecast, development of new products and new technology: A Study on product platform, support staff collaborative research, technology research and product development.

The research and application of enterprise knowledge network system, realize the intellectual assets in complex product development of effective incentives, improve efficiency, reduce duplication of investment, enhance the competitiveness of enterprises, and ultimately enable enterprises to achieve significant economic and social benefits.

\section{Top Level Design of Knowledge Network}

As a complex system engineering, the construction of enterprise knowledge network should first carry out the top-level design, carding the framework of the system. From the content point of view, the construction of knowledge network should be supported by the enterprise rules and regulations, operational standards and so on. Cry broken knowledge semantic expression, knowledge acquisition, rational self-organization and order and data integration technology of heterogeneous knowledge bottleneck, can make the knowledge network play a role; from the time dimension, establishment, operation and orderly, integrated business enterprise knowledge network of knowledge network.

The main work of the top-level design of enterprise knowledge network establishment process includes: the system design of enterprise knowledge network: sharing knowledge network requirements will be in the hands of personal knowledge resources between individuals and departments of mutual exchange of needed products, breaking the barriers of interest. This inevitably involves individuals or departments in the enterprise interests, so it is necessary to have the security system, established to promote knowledge network, promote enterprise innovation, improve the production efficiency, but also guarantee the establishment of knowledge (sharing) rights. For example, enterprises for employees sharing knowledge of intellectual property identification and evaluation, individual knowledge into organizational knowledge, the externalization of tacit knowledge into explicit knowledge at the same time, the staff contribution and performance appraisal linked to promote full collaborative innovation. The system involved here is: enterprise knowledge contribution assessment system, internal intellectual property system, etc.. In addition, enterprises can also promote the circulation of knowledge by means of knowledge borrowing and even by means of knowledge purchase.

The establishment of knowledge network standard design: the past enterprise has accumulated a lot of knowledge but often structured degree is low, difficult to directly in the computer system storage and application use, need to make it through the artificial text description and graphical model, 
standardization, support computer understanding. In order to improve the ability of knowledge reuse, it is necessary to pay a great deal of manpower cost. In order to avoid the occurrence of such problems, the establishment of knowledge network should make the perfect rules and constraints, to improve the effectiveness of knowledge network establishment and use: the establishment of knowledge document template standards, knowledge description standards, knowledge modeling, knowledge acquisition standard template, to facilitate automatic recognition and understanding of knowledge document; formulate domain terminology ontology the establishment of standards, ontology semantic mapping standards, important knowledge through semantic mapping, realize knowledge inference directly help enterprise decision.

The establishment of knowledge network platform design: system of knowledge network should be established a clear system platform, the knowledge network for complex product development process should be a system platform to support business knowledge, tool knowledge, and expert knowledge sharing and integration application. Key technology research of knowledge network in the establishment: for example: knowledge network dynamic hierarchy modeling, ontology construction, ontology mapping and storage based on the knowledge representation, knowledge oriented network knowledge acquisition technology.

\section{Top Level Design of Knowledge Network Operation and Ordering}

The manufacturing enterprise knowledge sources, on the same object knowledge description will often have multiple versions, but also filled with a lot of useless, failure, or even false knowledge content, knowledge of ordering degree is low, it is difficult to effectively use. For example, the enterprise patent database, the number of enterprises in the patent is huge, but the overall there are a lot of low quality, low value or no value of the patent term, confusion, enterprises need to rely on special maintenance, screening to identify. Haier group for the purpose of the establishment of the Ministry of intellectual property rights, in order to manage corporate patent applications while helping $\mathrm{R} \& \mathrm{D}$ personnel to select the necessary patent data. Enterprises should be reasonable means to sort out the long-term use of $\mathrm{R} \& \mathrm{D}$ personnel, and to guide the innovation and protection of patents and other important data to make it orderly, improve their utilization efficiency.

Therefore, the knowledge network should be considered in the design of the top-level design, including: the order of knowledge value: the evaluation value of knowledge value. It is necessary to determine the evaluation criteria, evaluation methods, and so on. The order of the knowledge relationship: the evaluation value of the relationship between knowledge. It is necessary to determine the evaluation criteria, the model of knowledge relationship standards, evaluation methods, etc..

The main contents of the top-level design of knowledge:

The system design of knowledge network operation and orderly: through the design of the system and requirements to encourage the personnel participating in the evaluation of knowledge application knowledge, knowledge through reasonable evaluation system to promote knowledge network orderly, such as employee collaborative knowledge evaluation system, knowledge network orderly contribution performance reward system can better play a role.

The standard design of knowledge network operation and order: in the process of knowledge network ordering, it is necessary to establish the standard of knowledge relation model, the description of knowledge relation, the knowledge value standard, the knowledge evaluation standard and so on.

Knowledge of network operation and platform design ordering: knowledge network ordering often need to rely on the power of the public, so the knowledge network ordering platform is an open platform, can let the public participate in, and to the public to participate in the process of tracking, recording, statistics and analysis, in order to encourage the enthusiasm of public participation and orderly work the. Knowledge network platform should be a platform to support collaborative evaluation and construction.

The key technologies in the process of knowledge network ordering are as follows: the knowledge evaluation technology based on the daily business, the knowledge based on the public participation and the collaborative evaluation technology. 
After breaking the barrier of knowledge sharing, knowledge integration should be taken as the main line of product life cycle of product design, production and service for manufacturing enterprises. Knowledge integration is not easy, there are technical reasons, such as the problem of heterogeneous data; and more management problems, such as in the business sector itself will be knowledge, information is not clear, because of concerns about information security or technical secrets, within the Department of even barriers of knowledge sharing. The most typical example: in the manufacture of military units, for information security, design and production experience of knowledge between different types of the same product is also hard to exchange, and even cut off; in addition to ensure between different departments, professional business together is difficult; therefore the knowledge network integrated application of top-level design major the content includes: the system design of knowledge network integration: between business enterprises to break the barriers, from the angle of management to develop business collaborative management system, ensure the knowledge interoperability between the corporate sector, professional services. The design of knowledge network integration standards: to establish a variety of standards for the integration of knowledge networks, mainly for the description and implementation of business processes. The design of knowledge network integration platform is to support the integration and transmission of heterogeneous knowledge between different departments, and to support the integration of different levels of knowledge from the bottom to the top. Integration platform not only to support the management of the integration, planning and decision-making, but also to help the business department to acquire the knowledge and experience of internal and external enterprises. For example, the business department of the input and output of product design results include design skills and a lot of experience in the business sector, if the design process, experience related skills are also transferred to the downstream business, can help other departments to quickly understand the design intent, the design process of rapid decision making. Enterprise managers can also clear the technical advantages and disadvantages of the enterprise from the knowledge network, help enterprises to plan the strategic direction and make reasonable product planning and production plan. Knowledge network platform should be a platform to support professional business process integration.

For the complex product design process, the knowledge network platform should also be able to support the integration of design tools (software). Because of the product design process, the business sector, a lot of knowledge and experience skills are integrated into the design tools. If you can integrate the professional business processes and the integration of design tools, the knowledge network platform will play a greater value. Therefore, the knowledge network platform should be a platform that can support the integration of professional tools. The key technologies of knowledge network integration include: heterogeneous data integration technology, design tools integration technology, business process knowledge integration technology, knowledge network information security technology and so on.

\section{Construction Process of Knowledge Network}

First of all, in the construction of enterprise knowledge networks, the need for the greatest degree of abstraction of the knowledge content, the relationship between knowledge, knowledge and knowledge by description of the limited expression, to ensure the consistency of knowledge expression. After that, the construction of the knowledge base of the knowledge network is considered, which mainly involves the acquisition of knowledge and the establishment of the network relation. For knowledge acquisition should be based on the characteristics of knowledge, considering different methods to obtain the explicit knowledge and the tacit knowledge to explicit knowledge respectively; the main consideration should be the internal and external knowledge, structural knowledge and unstructured knowledge, theoretical knowledge and practical knowledge of these heterogeneous knowledge should be flexible and effective control for the acquisition of tacit knowledge; that should be considered if the maximum promote the explicit encoding processing. It should be noted that in the process of knowledge acquisition, we must realize the unification of knowledge description model in knowledge network. For the establishment of knowledge network relationship, we should start from the characteristics of knowledge network, 
consider the knowledge of the situation, the main body and other aspects of the elements, from a dynamic multi-level perspective to build a knowledge network.

The operation of the knowledge network should be combined with the business of the enterprise, so it is necessary to realize the integration of knowledge push. In addition, due to the dynamic characteristics of the knowledge network, the construction of knowledge network can not be achieved in one step, nor can it be done once and for all. Therefore, the design of knowledge network should ensure that it has the function of self-organization optimization in the process of running, and can realize the feedback of the knowledge network operation with the user's daily work behavior. On the one hand, knowledge evaluation value of knowledge, knowledge of the implementation of The new supersedes the old. network, on the other hand through the feedback and interaction to promote the birth of new knowledge (including the acquisition of new knowledge and new knowledge creation and demand).

Knowledge modeling is the premise and basis of knowledge of network construction, the comprehensive expression of knowledge network in single point data of heterogeneous knowledge, knowledge abstraction, knowledge structure to form the minimum element attribute and element attribute constraints and element attribute mapping knowledge item description unit.

A dynamic knowledge network model based on a unified knowledge knowledge is the core technology of network construction, it is not the knowledge modeling repetition, but from a deeper knowledge of the relationship between knowledge integration and application conditions are analyzed and described. This paper analyzes the various states of knowledge from the aspects of knowledge subject, situation and value. On this basis, the knowledge network model is constructed, which includes hierarchical network model and dynamic evolutionary model. When the two models are completed, the construction of knowledge network has begun to take shape.

\section{Acknowledgement}

This work was supported by the planning project on culture and art of Jiangxi province (YG2016034).

\section{References}

[1] Biichel B, Raub S. Building knowledge-creating value networks. European Management Journal, Vol.6.2002, pp.587-596.

[2] Cannataro M, Talia D. The knowledge grid. Communications of the ACM, Vol.46.2003, pp.89-93.

[3] BRIN S, PAGE L. The anatomy of a large-scale hypertextual Web search engine. Computer Networks and ISDN Systems, Vol.30.1998,pp.107-117.

[4] Marakas G M. Decision support systems in the 21st century. Upper Saddle River, NJ: Prentice Hall, 2003.

[5] Lee H J, Cho K, et al. Utilizing knowledge context in virtual collaborative work. Decision Support Systems, Vol.39.2005, pp.563-582. 